\title{
PIASy is a SUMOylation-independent negative regulator of the insulin transactivator MafA
}

\author{
Suzuka Onishi and Kohsuke Kataoka \\ Laboratory of Molecular Medical Bioscience, Graduate School of Medical Life Science, Yokohama City University, Yokohama, Japan \\ Correspondence should be addressed to K Kataoka: kkataoka@yokohama-cu.ac.jp
}

\begin{abstract}
Insulin plays a central role in glucose homeostasis and is produced exclusively by pancreatic islet $\beta$-cells. Insulin gene transcription is regulated by a set of $\beta$-cell-enriched transcription factors that bind to cis-regulatory elements within the promoter region, and regulation of the insulin gene promoter is closely linked to $\beta$-cell functionality. PIASy, a member of the PIAS family of SUMO E3 ligases, is thought to affect insulin gene transcription, but its mechanism of action is not fully understood. Here, we demonstrate that PIASy interacts with MafA and represses insulin gene promoter activity. MafA is a $\beta$-cell-restricted basic leucine-zipper transcriptional activator that binds to the $\mathrm{C} 1$ element of the insulin gene promoter. In line with previous studies showing the transactivator domain of MafA is SUMOylated, PIASy enhanced the SUMOylation of MafA. However, a SUMOylation-deficient mutant of MafA was still repressed by PIASy, indicating that this modification is dispensable for repression. Using a series of MafA and PIASy mutants, we found that the basic domain of MafA and the amino-terminal region of PIASy containing the SAP domain are necessary for their interaction. In addition, SUMO-interacting motif 1 (SIM1) at the carboxyl-terminal region of PIASy was required to repress the synergistic transactivation of MafA, $\mathrm{Pdx} 1$, and Beta2, transcription factors playing central roles in $\beta$-cell differentiation and function. The PINIT and SP-RING domains in the middle region of PIASy were dispensable. These findings suggest that PIASy binds to MafA through the SAP domain and negatively regulates the insulin gene promoter through a novel SIM1dependent mechanism.
\end{abstract}



Journal of Molecular Endocrinology (2019) 63, 297-308

\section{Introduction}

Insulin is a peptide hormone secreted from pancreatic $\beta$-cells that plays a critical role in glucose homeostasis. The $\beta$-cell-restricted expression of the insulin (preproinsulin) gene is directed by its $\sim 350 \mathrm{bp}$ promoter region and several $\beta$-cell-enriched transcription factors, which bind to evolutionally conserved cis-regulatory elements within the promoter (Fig. 2A). Accumulating evidence has indicated that three transcription factors, Pdx1, MafA, and Beta2, play central roles in controlling expression of the insulin gene (Wilson et al. 2003, Bernardo et al. 2008). Pdx1 binds to the A1, A3/A4 and GG2 elements of the promoter, whereas MafA and Beta2 bind to the $\mathrm{C} 1$ and $\mathrm{E} 1$ elements, respectively, physically interact with each other, and synergistically activate the promoter (Aramata et al. 2005, Docherty et al. 2005, Kaneto et al. 2005, Han et al. 2016). Additional transcription factors such as Islet1 and Glis3 also bind to the A3/A4 and GlisBS elements, respectively, and activate the promoter in cooperation with the core 
transcription factors Pdx1, MafA, and Beta2 (Yang et al. 2009, Zhang et al. 2009, ZeRuth et al. 2013, Jung et al. 2018). These $\beta$-cell-enriched transcription factors are also involved in the expression of other $\beta$-cell-specific genes and in $\beta$-cell function and differentiation. For example, MafA expression begins at the latest stage of the $\beta$-cell differentiation process and is essential for acquisition of the mature cellular phenotype, in particular, the ability to secrete insulin in response to glucose (Nishimura et al. 2006, Artner et al. 2010). Ablation of the $P d x 1$ or Beta2 gene in mice results in impaired $\beta$-cell differentiation and a severe diabetic phenotype (Naya et al. 1997, Fujitani et al. 2006, Gu et al. 2010). In humans, heterozygous mutations in the PDX1 and BETA2 genes are responsible for maturity-onset diabetes of the young (MODY) types 4 and 6, respectively (Stoffers et al. 1998, Malecki et al. 1999). Furthermore, because ectopic expression of appropriate combination of lineage-restricted transcription factors can lead to cellular reprogramming, expression of Pdx1, MafA, and Beta2 (or Ngn3) in pancreatic exocrine or other cell types leads to $\beta$-cell transdifferentiation (Zhou et al. 2008, Chen et al. 2014). In particular, ectopic expression of Pdx1 and MafA in islet $\alpha$-cells induces conversion into $\beta$-cells (Furuyama et al. 2019). These $\beta$-cell reprogramming technologies are promising tools to treat diabetes.

Insulin gene transcription is affected by various intracellular and extracellular conditions. For instance, oxidative stress downregulates $\operatorname{Pdx} 1$ and MafA, thereby decreasing insulin expression and compromising $\beta$-cell function (Robertson 2004, Harmon et al. 2005). During the progression of diabetes, prolonged high glucose conditions reduce the abundance of MafA protein, resulting in a decrease in insulin expression (Butler et al. 2012, Guo et al. 2013). Thus, elucidating the regulatory mechanisms of transcription factors involved in insulin gene expression will aid our understanding of $\beta$-cell pathophysiology and contribute to the development of $\beta$-cell-regenerative therapies.

Previous reports suggested that PIASy, a member of PIAS family of small ubiquitin-like modifier (SUMO) E3 ligases, affects the activities of the Islet1 and Glis3 transcription factors (Yan et al. 2016, Hoard et al. 2018). SUMO is covalently conjugated to the lysine residues of target proteins through the SUMO-specific conjugating enzyme Ubc9 and E3 ligases. The biological consequences of SUMO conjugation to target proteins are diverse, and in many, but not all cases involving transcription factors, SUMO modification represses transcriptional activity. Several PIASy-interacting proteins have been identified to date, and the SUMOylation of most of them is stimulated by PIASy. For example, PIASy interacts with the Smad3 and Lef1 transcription factors, enhances their SUMOylation and represses their transcriptional activities (Sachdev et al. 2001, Long et al. 2003). By contrast, PIASy represses androgen receptor activity in a manner that is independent of SUMOylation but dependent on the recruitment of histone deacetylase HDAC1/2 through its amino-terminal domain (amino acids 1-90) (Gross et al. 2004). Therefore, PIASy affects transcription through multiple mechanisms.

PIASy contains several functional domains, namely the SAP, PINIT, and SP-RING domains (Fig. 5C). The amino-terminal SAP domain is able to bind AT-rich DNA sequences and promotes association of PIASy with the nuclear matrix region (Sachdev et al. 2001). The PINIT domain is involved in the subcellular localization of PIASy and recognition of some substrates (Duval et al. 2003). The SP-RING domain resembles the RING finger motif of many ubiquitin E3 ligases and associates with the SUMOconjugating enzyme Ubc9 (Joazeiro \& Weissman 2000). Thus, the SP-RING domain is necessary for the SUMO conjugation of many substrates; however, in some cases, this domain is dispensable for SUMOylation. For example, PIASy stimulates SUMOylation of Yin Yang1 protein independent of the SP-RING domain (Deng et al. 2007). The carboxyl-terminal region of PIASy also contains two SUMO-interacting motifs (SIM1 and SIM2), which mediate non-covalent SUMO binding (Rytinki et al. 2009, Kaur et al. 2017).

A previous study demonstrated that PIASy associates with Glis3, stimulates its SUMOylation, and represses its transcriptional activity (Hoard et al. 2018). PIASy also interacts with Islet1 and stimulates its transcriptional activity (Yan et al. 2016); however, it is not known whether PIASy stimulates Islet1 SUMOylation. Despite these findings, the role of PIASy in the regulation of insulin gene transcription remains elusive. Furthermore, MafA is SUMOylated at Lys32 within its amino-terminal transactivator domain, and this modification negatively regulates its transcriptional activity (Shao \& Cobb 2009, Kanai et al. 2010). The possible involvement of PIAS proteins in the SUMOylation of MafA is also not known.

In this report, we demonstrate that PIASy is a negative regulator of the insulin gene promoter and characterize the molecular mechanisms involved in this regulation. We found that PIASy physically interacts with MafA through the SAP domain, enhances its SUMOylation, and suppresses its transactivator function in a SUMOylationindependent manner. We also found that the PINIT and SP-RING domains of PIASy are dispensable for suppressing 
the synergistic action of MafA, Pdx1, and Beta2, whereas the SAP domain and SIM1 are required. These observations suggest a novel mechanism of transcriptional repression by PIASy.

\section{Materials and methods}

\section{Plasmids}

The expression plasmids harboring shRNAs targeting PIASy (pEF3-GFPBsr-U6/PIASy \#1 and \#2) were constructed by introducing double-stranded oligonucleotides into the BseRI-BglII restriction sites of the pEF3-GFPBsr-U6 vector, as described previously (Kanai et al. 2011). The target sequences of shRNAs \#1 and \#2 were 5'-CUACAGAGGU UGAAGACGA-3' and 5'-GAUGAGCUUCCGAGUAUCA-3', respectively.

The firefly luciferase reporter plasmids pGL4/h-ins-p was constructed by inserting the KpnI-HindIII fragments of the pGL2-based plasmids h-insp-luc (Aramata et al. 2005) into pGL4.10 (Promega). The pGL4/core-RBGP was constructed by inserting the KpnIHindIII fragments of the pGL2-based core-enh/TATA-luc (Han et al. 2011) containing the core enhancer region of the insulin gene promoter and the TATA-box and the transcription start site of the rabbit $\beta$-globin gene promoter (RBGP, GenBank:V00878.1) into pGL4.10. The pGL4/h-ins-p ( $\Delta$ BglII) plasmid was constructed by digestion of pGL4/h-ins-p with BglII and KpnI, followed by blunting and self-ligation. Mutations of the GG2, C1, and E1 elements have been described previously (Kataoka et al. 2002, Aramata et al. 2005). The GlisBS (5'-CCCCCCCACCCC-3') and A1 (5'-CCTAATGG-3') elements were mutated to 5'-CagtCCgACCtC-3' and 5'-CCTAcTGG-3', respectively, by site-directed overhang extension PCR mutagenesis. To construct the pGL4/3xC1-RBGP plasmid, three tandem repeats of the C1-containing double-stranded oligonucleotide (5'-GATCCGGAAATTGCAGCCTCAGCCCCCAGCCA-3' and 5'-GATCTGGCTGGGGGCTGAGGCTGCAATTT CCG-3') and a double-stranded oligonucleotide spanning the TATA-box and the transcription start site of the rabbit $\beta$-globin gene promoter (RBGP) (5'-GATCTTGGGCA TAAAAGGCAGAGCACTGCAGCTGCTGCTTA-3' and 5' -AG CTTAA GCAGC AGCTG CAGTG CTCTG CCTTT TATGCCCAA-3') were inserted into the pGL4.10 plasmid. To construct pGL4/5xGAL4-RBGP, a DNA fragment containing five tandem repeats of the GAL4-upstream activating sequence was amplified from the pUAST plasmid (Brand \& Perrimon 1993) using the following primers: 5'-CGATAGGTACCGCATCTCTCCGGATCC AAGCTTGC-3' and 5'-TAAAAGATCTTATACTCCGGCG CTCGCTAGAGTCT-3'. The fragment was then inserted into the pGL4/3xC1-RBGP plasmid by replacing the KpnIBglII fragment containing the three tandem C1 elements. The expression plasmid for Renilla luciferase (pEF-Rluc) has been described previously (Kataoka et al. 2001a), and the pRL-TK plasmid was purchased from Promega.

The pCIdS expression vector was constructed from the pCI plasmid (Promega) by SpeI digestion, blunting, and self-ligation to eliminate the SpeI restriction site. To construct the expression plasmid harboring fulllength FLAG-tagged human PIASy (pCIdS/FLAGhPIASy), the entire open reading frame of human PIASy (NM_015897.4) was amplified by RT-PCR using the following primers: 5'-CGAGAATTCGCGCGCAT CGATAGGATCCACCATGGATTACAAGGATGACGAC GATAAGGGAACTAGTATGGCGGCGGAGCTGGTGG$3^{\prime}$ and 5'-CTGCTCGAAGCGGCCGCTAGCAGGCCG GCACCAGG-3'. The fragment was then inserted into the EcoRI-NotI site of the pCIdS vector. To construct pCIdS/HA-m-MafA, the NheI-NotI insert of the pCIdS/ FLAG-hPIASy plasmid was replaced by a XbaI-NotI fragment containingtheHA-taggedmouseMafAopen readingframeof pHygEF2/HA-m-MafA. The pHygEF2/HA-m-MafA (Han et al. 2007), pHygEF2/FLAF-Pdx1, pHygEF2/FLAG-Beta2 (Kataoka et al. 2002), pHygEF2/FLAG-SUMO1 (Kanai et al. 2010), pEF/Gal4-DBD, pEF/Ga4-VP16 (Kataoka et al. 2001b), and pEF/Gal4-MafA (Han et al. 2007) plasmids have been described previously. All other substitution, deletion, and fusion mutants were generated by PCR-based mutagenesis.

\section{Cells}

The mouse insulinoma-derived MIN6 cell line was a generous gift from Dr Jun-ich Miyazaki (Osaka University) (Miyazaki et al. 1990). The NIT-1 mouse insulinoma cell line was purchased from American Tissue Culture Collection. The human cervical carcinoma-derived HeLa cell line lacks expression of endogenous MafA, Pdx1, and Beta 2 and was used for reconstitution experiments. These cells were grown in Dulbecco's modified Eagle's medium supplemented with 15\% (MIN6) or 10\% (NIT-1 and HeLa) fetal calf serum.

\section{Luciferase assay}

MIN6 and NIT-1 cells were reverse-transfected with 1.8 or $1.6 \mu \mathrm{g}$ of plasmid using Lipofectamine 3000 or 
Lipofectamine 2000 reagent (Thermo Fisher Scientific), respectively, and seeded into 48-well plates. HeLa cells grown in 48 -well plates were transfected with $0.8 \mu \mathrm{g}$ of plasmid using Lipofectamine 2000 reagent. The cells were harvested $48 \mathrm{~h}$ (MIN6 and NIT-1) or $24 \mathrm{~h}$ (HeLa) after transfection, and firefly and Renilla luciferase activities were measured using a dual luciferase assay system (Promega) or the Twinlite Firefly and Renilla Luciferase Reporter Gene Assay System (Perkin Elmer). Firefly luciferase activities were normalized on the basis of Renilla luciferase activities, and data represent the mean \pm s.E. of at least three independent experiments.

\section{Immunoprecipitation and immunoblotting}

For immunoprecipitation, HeLa cells grown in six-well plates were transfected with $3.2 \mu \mathrm{g}$ of plasmid using $8 \mu \mathrm{L}$ of Lipofectamine 2000 reagent (Thermo Fisher Scientific). Whole cell extracts were prepared by cell lysis in $800 \mu \mathrm{L}$ of NETN buffer $(150 \mathrm{mM} \mathrm{NaCl}, 1 \mathrm{mM}$ EDTA, $10 \mathrm{mM}$ Tris- $\mathrm{HCl}$ (pH 8.0), and $0.05 \%$ Nonidet P-40) and sonication. Aliquots of the extracts were subjected to immunoprecipitation using anti-FLAG-agarose (SigmaAldrich), followed by immunoblotting using an anti-HA (MBL, Nagoya, Japan), anti-FLAG (Sigma-Aldrich), or antiGFP (Clontech Laboratories, Mountain View, CA, USA) antibody as described previously (Han et al. 2011).To detect SUMOylated MafA protein, HeLa cells grown in 24-well plates were transfected with $1.6 \mu \mathrm{g}$ of plasmid using $2 \mu \mathrm{L}$ of Lipofectamine 2000 reagent (Thermo Fisher Scientific). Whole cell extracts were prepared by the direct addition of $40 \mu \mathrm{L}$ of sodium dodecyl sulfate (SDS) sample buffer (200 mM of Tris-HCl, pH 6.8, 8\% SDS, $400 \mathrm{mM}$ dithiothreitol, $0.2 \%$ bromophenol blue, and $40 \%$ glycerol) to the cells.

\section{Results}

\section{PIASy is a negative regulator of insulin gene promoter activity}

To investigate the role of PIASy in the regulation of insulin gene expression in $\beta$-cells, we carried out a luciferase reporter gene assay using two different mouse insulinoma-derived cell lines, MIN6 and NIT-1. These cells were co-transfected with a luciferase reporter plasmid driven by the human insulin gene promoter (pGL4/h-ins-p, Fig. 2A) and an expression vector harboring one of two different shRNAs targeting PIASy. Unfortunately, the transfection efficiencies of these cell

lines are too low to estimate the knockdown efficacy. However, the cells receive the shRNA expression vector and the reporter plasmids simultaneously and thus the effect of the PIASy knockdown can be evaluated qualitatively. In both cell lines, the PIASy-targeting shRNAs increased the luciferase activity (Fig. 1A and B), indicating that the activity of the insulin gene promoter is negatively regulated by endogenous PIASy. Conversely, co-transfection of an expression vector harboring PIASy significantly reduced the luciferase activity (Fig. 1C). By contrast, overexpression of other PIAS family members,

\section{A MIN6}
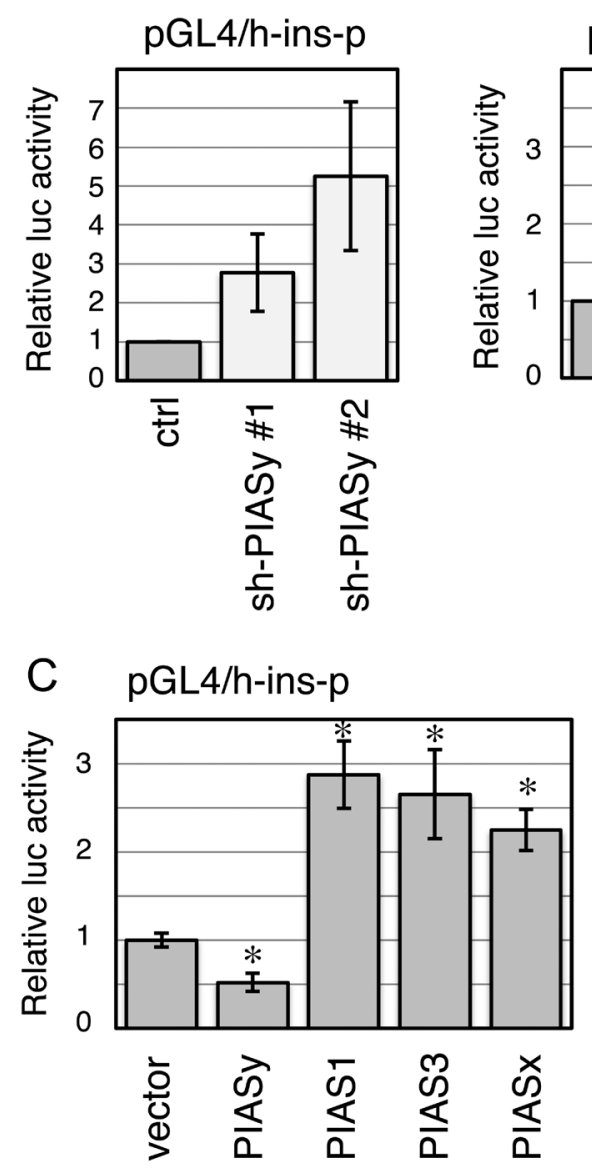

Figure 1

PIASy is a negative regulator of the insulin gene promoter. ( $A$ and $B$ ) The mouse insulinoma-derived MIN6 (A) and NIT-1 (B) cell lines were transfected with the pGL4/h-ins-p firefly reporter plasmid, the pEF-Rluc Renilla reporter plasmid, and an expression plasmid harboring the indicated shRNA. Data are expressed relative to the luciferase activity in cells transfected with the control shRNA expression vector, and represent the mean \pm S.E. of three independent experiments. (C) NIT-1 cells were transfected with $0.25 \mu \mathrm{g}$ of pGL4/h-ins-p, $0.25 \mu \mathrm{g}$ of pRL-TK, and $1.1 \mu \mathrm{g}$ of expression plasmid harboring the indicated PIAS protein. Data are expressed relative to the luciferase activity in cells transfected with the empty expression vector. Data represent the mean \pm S.E. of six independent experiments. ${ }^{*} P<0.05$ (Student's $t$ test).

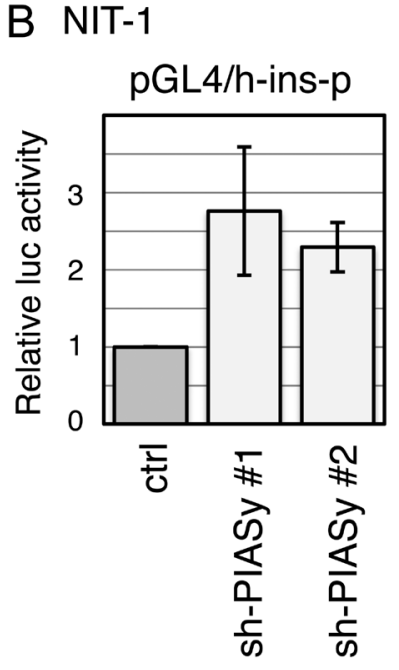


A

pGL4/h-ins-p (FL)

pGL4/h-ins-p ( $\Delta$ Bg III)

pGL4/core-RBGP
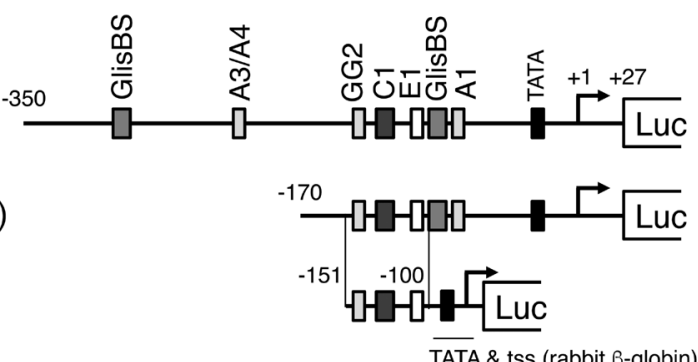

B

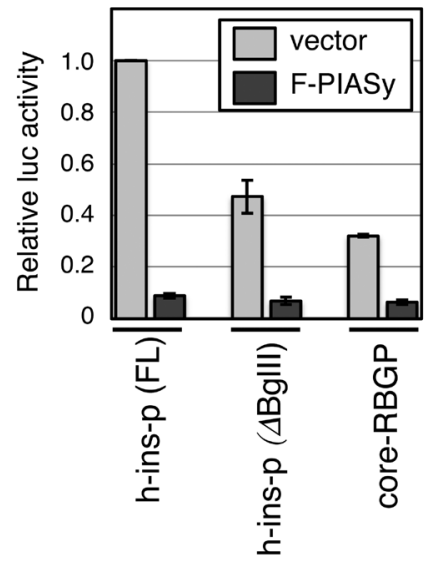

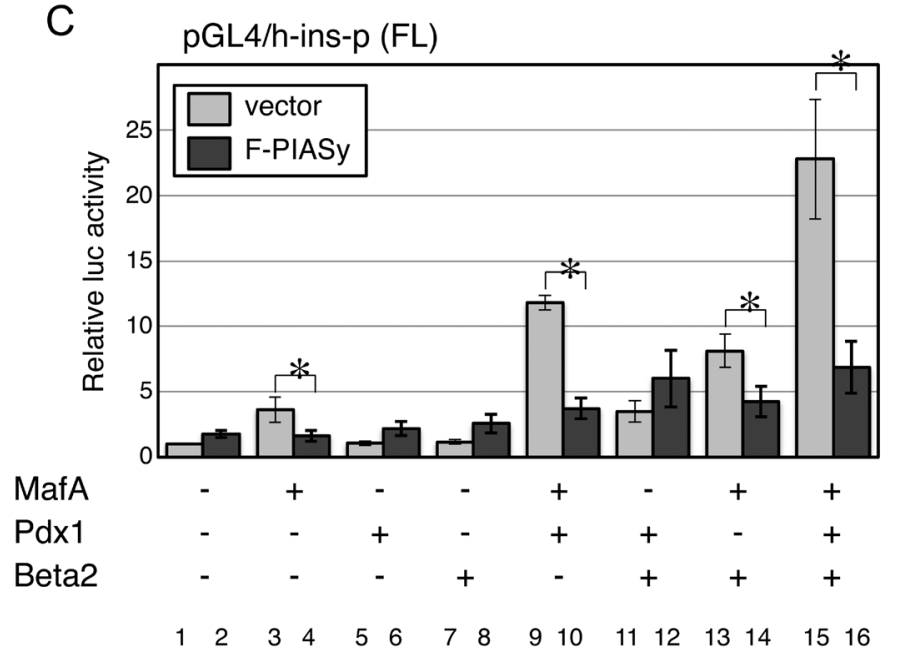

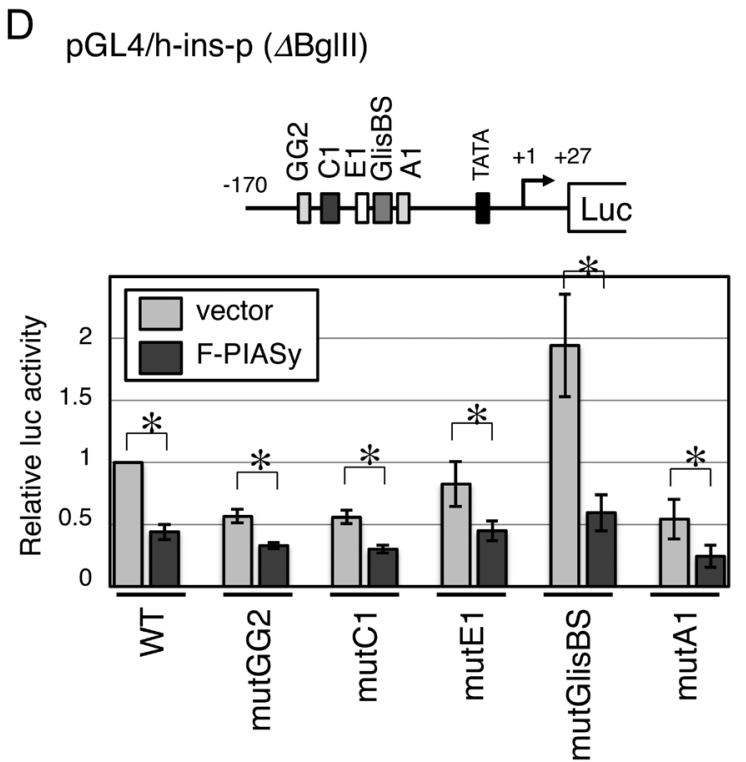

\section{Figure 2}

PIASy represses the activity of the insulin gene promoter in the presence of MafA. (A) Schematic representations of the promoter constructs used in this study. The locations of the binding sites for Pdx1 (A1, A3/A4, and GG2), MafA (C1), Beta2 (E1), Islet1 (A3/A4), and Glis3 (GlisBS) are indicated. (B) NIT-1 cells were transfected with $0.25 \mu \mathrm{g}$ of the indicated reporter plasmid, $0.25 \mu \mathrm{g}$ of pRL-TK, and $1.3 \mu \mathrm{g}$ of pCIdS empty vector or pCIdS/FLAG-hPIASy plasmid. Data are expressed relative to the activity in cells transfected with $p G L 4 / h-i n s-p(F L)$ and the empty expression vector and represent the mean \pm s.E. of three independent experiments. (C) HeLa cells were transfected with $0.1 \mu \mathrm{g}$ of pGL4/h-ins-p, $0.1 \mu \mathrm{g}$ of pRL-TK, various combinations of the indicated plasmids $(0.16 \mu \mathrm{g}$ each), and the FLAG-tagged PIASy expression plasmid or empty vector $(0.12 \mu \mathrm{g})$. Data represent the mean \pm S.E. of four independent biological replicates. (D) The firefly luciferase reporter plasmid pGL4/h-ins-p $\Delta$ Bglll or its derivatives $(0.25 \mu \mathrm{g})$ and pRL-TK $(0.25 \mu \mathrm{g})$ were transfected into NIT-1 cells with or without the FLAG-PIASy expression plasmid $(1.3 \mu \mathrm{g})$. Data represent the mean \pm S.E. of four to nine independent biological replicates. $* P<0.05$ (Student's $t$ test).

namely PIAS1, PIAS3, or PIASx, rather increased the luciferase activity. Thus, among PIAS family members, PIASy is a negative regulator of insulin gene promoter activity in $\beta$-cells.

\section{PIASy represses the transcriptional activity of MafA}

The insulin gene promoter contains multiple cisregulatory elements that are bound by various transcription factors (Fig. 2A), and the core region spanning the GG2, C1, and E1 elements plays central and essential roles in controlling the $\beta$-cell-restricted expression of the insulin gene (Bernardo et al. 2008). To understand the mechanism of PIASy-mediated repression of the insulin gene promoter activity, we constructed a reporter plasmid that lacked the $5^{\prime}$ distal region but retained the core and proximal regions (pGL4/h-ins-p $\Delta$ BglII) (Fig. 2A). Although this promoter 
construct exhibited lower luciferase activity than the full-length (FL) promoter reporter in NIT-1 cells, expression of PIASy reduced its activity (Fig. 2B). Subsequently, we constructed a reporter that contained only the core elements (GG2, C1, and E1) of the insulin gene promoter, along with the heterologous TATAbox and transcription start site of the rabbit $\beta$-globin promoter (RBGP) region (pGL4/core-RBGP) (Fig. 2A), and found that PIASy again repressed the reporter activity (Fig. 2B). This finding indicates that the core region of the insulin gene promoter spanning the GG2, C1, and E1 elements is sufficient for PIASy-mediated repression.

As the GG2, C1, and E1 elements are bound by transcription factors Pdx1, MafA, and Beta2, respectively, we attempted to determine the factor(s) responsible for PIASy-mediated repression of the insulin gene promoter. To this end, we utilized a reconstitution system using HeLa cells, a non- $\beta$ cell line that does not express endogenous Pdx1, MafA, or Beta2. As reported previously (Aramata et al. 2005), exogenous expression of MafA, Pdx1, or Beta2 alone had only a marginal effect on the activity of the insulin promoter reporter in HeLa cells (Fig. 2C, lanes 1, 3, 5 , and 7), whereas combinations of two or three of these factors resulted in an additive or synergistic increase in the reporter activity (Fig. 2C, lanes 9, 11, 13, and 15). In line with the observed reduction in endogenous promoter activity by overexpression of PIASy in NIT-1 cells (Fig. 2A), co-expression of PIASy reduced the synergistic activity of Pdx1, MafA, and Beta2 in HeLa cells (Fig. 2C, lane 16). PIASy repressed the luciferase activity in HeLa cells in the presence of MafA (Fig. 2C, lanes 4, 10, and 14), but not in its absence (Fig. 2C, lanes 6, 8, and 12). These observations indicate that PIASy represses the insulin gene promoter via MafA-dependent mechanisms.

To determine whether MafA is the sole transcription factor that mediates PIASy repression of the insulin gene promoter in $\beta$-cells, we constructed a series of $5^{\prime}$ truncated pGL4/h-ins-p $\Delta$ BglII reporter plasmids with mutations of the cis-regulatory elements, GG2, C1, E1, GlisBS, and A1 (Fig. 2D) and evaluated their activities in the absence or presence of exogenous PIASy in NIT-1 cells. Mutation of the $\mathrm{C} 1$ element reduced the basal activity of the luciferase reporter, and its activity was reduced further by co-expression of PIASy (Fig. 2D). Reporter plasmids in which the GG2, E1, GlisBS, or A1 element was mutated and were also responsive to PIASy (Fig. 2D). These results indicate that PIASy targets multiple transcription factors and cis-elements to repress the activity of the insulin gene promoter in $\beta$-cells and suggest that MafA is one of the targets.

\section{PIASy binds to MafA and enhances its SUMOylation}

To further establish the roles of MafA in transcriptional repression of the insulin gene promoter by PIASy, we investigated whether PIASy physically interacts with MafA. To this end, HA-tagged MafA and FLAG-tagged PIASy were co-expressed in HeLa cells, and the cell extracts were subjected to immunprecipitation using an antiFLAG antibody. Immunoblotting revealed that HA-MafA was co-immunoprecipitated with FLAG-PIASy (Fig. 3A), indicating a physical interaction between the proteins.

MafA is reportedly SUMOylated at Lys32 (K32) in $\beta$-cells (Shao \& Cobb 2009, Kanai et al. 2010), and PIASy
A



$B$

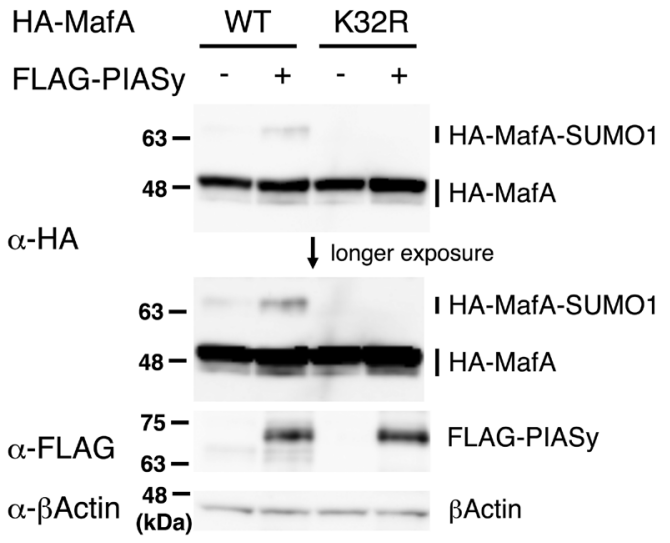

Figure 3

PIASy stimulates MafA SUMOylation. (A) HA-tagged MafA was co-expressed with or without FLAG-tagged PIASy in HeLa cells. The cell extracts were subjected to immunoprecipitation with an anti-FLAG antibody, and the immune complexes ( $\alpha$-FLAG I.P.) were analyzed by immunoblotting with an anti-HA or anti-FLAG antibody. As a control, total cell extracts (input) were also analyzed. IgH, immunoglobulin heavy chain. The data are representative of two independent biological replicates. (B) HA-tagged wild-type (WT) MafA or the K32R mutant was co-expressed in HeLa cells with FLAG-tagged SUMO1 and with or without FLAG-tagged PIASy. Total cell extracts were subjected to immunoblotting with an anti-HA, anti-FLAG, or anti- $\beta$-actin antibody. Signals corresponding to HA-MafA and its SUMOylated forms (HA-MafASUMO1) are indicated. The data are representative of two independent experiments. 
is a member of the SUMO E3 family of ligases. Therefore, we examined whether PIASy affects MafA SUMOylation. HeLa cells were transfected with expression plasmids harboring HA-tagged MafA and SUMO1, and the whole cell lysates were subjected to immunoblot analysis (Fig. 3B). An anti-HA antibody detected the HA-MafA protein as multiple bands of approximately $50 \mathrm{kDa}$, as well as slower migrating forms of approximately $65 \mathrm{kDa}$. The latter bands were not observed in cells transfected with the HA-tagged MafA K32R mutant, in which Lys32 was substituted with Arg, indicating that they corresponded to the SUMOylated form of MafA, as reported previously (Kanai et al. 2010). Co-expression of FLAG-tagged PIASy in the transfected cells increased the amount of the SUMOylated MafA, indicating that PIASy enhances the SUMOylation of MafA.

\section{SUMOylation of MafA is dispensable for its repression by PIASy}

Enhancement of SUMO conjugation is a common mechanism by which PIAS family proteins represses the transcriptional activities of their targets. Therefore, we examined whether PIASy represses MafA activity by enhancing its SUMOylation. In a reconstitution system using HeLa cells, both wild-type MafA and the SUMOylation-deficient K32R mutant activated the insulin gene promoter synergistically with Pdx1 and Beta2 and both activities were inhibited by overexpression of PIASy (Fig. 4A). This finding indicates that SUMOylation of MafA is not required for repression by PIASy.

Next, we examined whether PIASy affects the function of the transactivation domain of MafA using a Gal4-fusion system. MafA has a basic leucine zipper (bZip) domain at its carboxyl-terminal that acts as the DNA-binding domain, whereas the transactivator domains are assigned to its amino-terminal region (Fig. 4B, upper panel). The aminoterminal region (MafA-N) was fused to the DNA-binding domain of the yeast transcription factor Gal4 (Gal4DBD). The resultant fusion protein (Gal4-MafA) induced reporter activity of a plasmid containing tandem copies of the Gal4-binding site, and co-expression of PIASy did not affect this activity (Fig. 4B, lower panel). The activity of a fusion construct comprising the Gal4-DBD linked to the
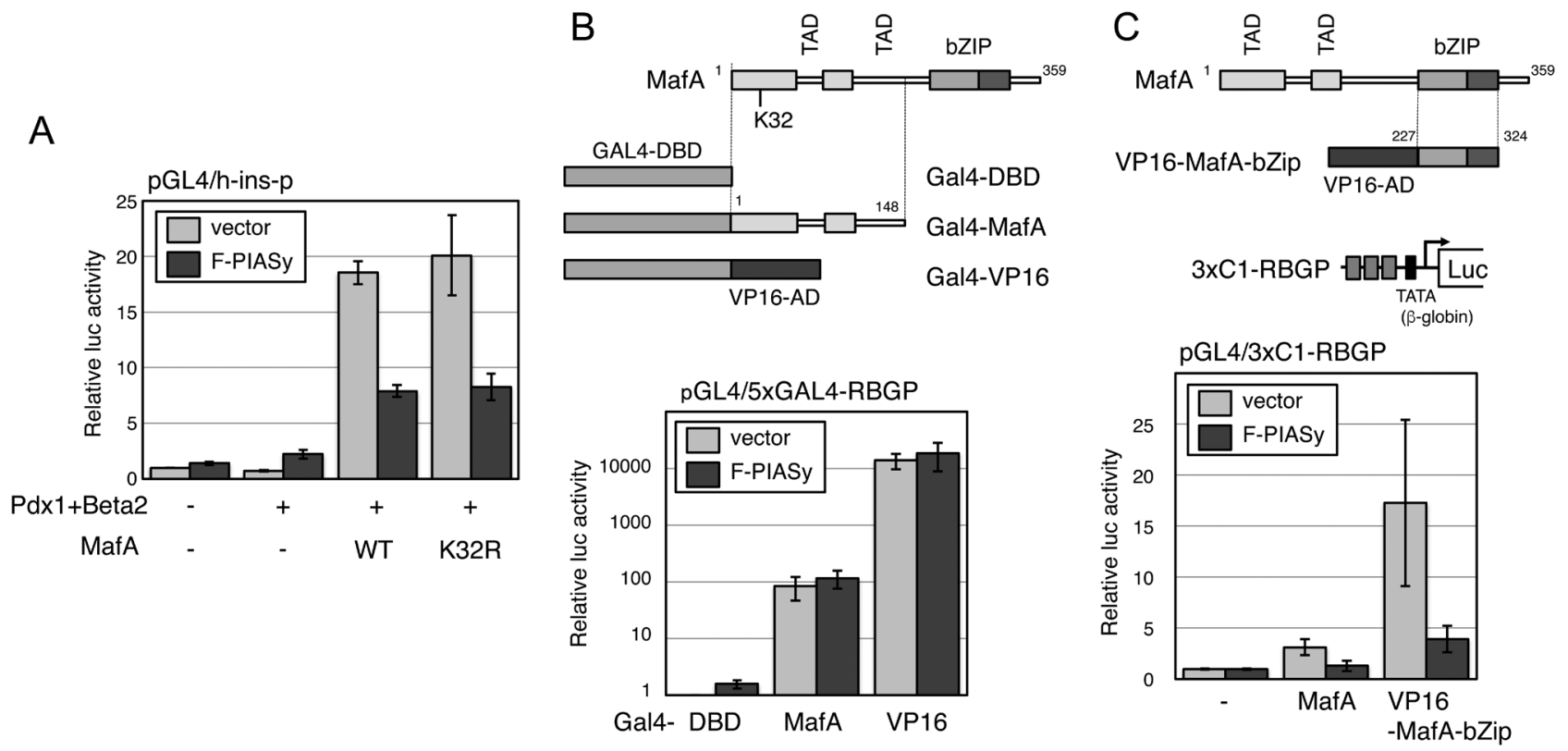

\section{Figure 4}

PIASy represses MafA activity in a manner that is independent of SUMOylation and dependent on the bZip domain. (A) HeLa cells were transfected with $0.1 \mu \mathrm{g}$ of pGL4/h-ins-p, $0.1 \mu \mathrm{g}$ of pRL-TK, the indicated combinations of expression plasmids harboring Pdx1, Beta2, and wild-type (WT) MafA or the K32R mutant $(0.16 \mu \mathrm{g}$ each), and with or without FLAG-tagged PIASy $(0.12 \mu \mathrm{g})$. Data are expressed relative to the luciferase activities of pGL4/h-ins-p-luc and empty vectors. Data represent the mean \pm s.E. of four independent biological replicates. (B) A reporter plasmid containing multiple Gal4-binding sites (pGL4/5xGAL4-RBGP) and expression plasmids harboring the indicated Gal4 DNA-binding domain (GAL4-DBD) fusion proteins (see upper panels) were transfected into HeLa cells with or without the FLAG-tagged PIASy expression plasmid. Data are expressed relative to the activity in cells transfected with GAL4-DBD and the empty expression vector and represent the mean \pm S.E. of three independent experiments. (C) HeLa cells were transfected with a luciferase reporter plasmid containing three copies of the insulin gene promoter C1 element (pGL4/3xC1-RBGP) and an expression vector harboring HA-tagged MafA or a VP16-MafA fusion protein (VP16-MafA-bZip), and with or without the PIASy expression vector. Data represent the mean \pm s.E. of four independent experiments. 
A

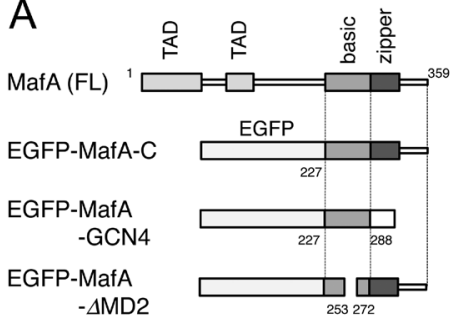

C

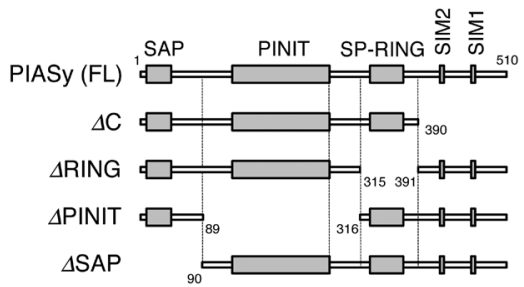

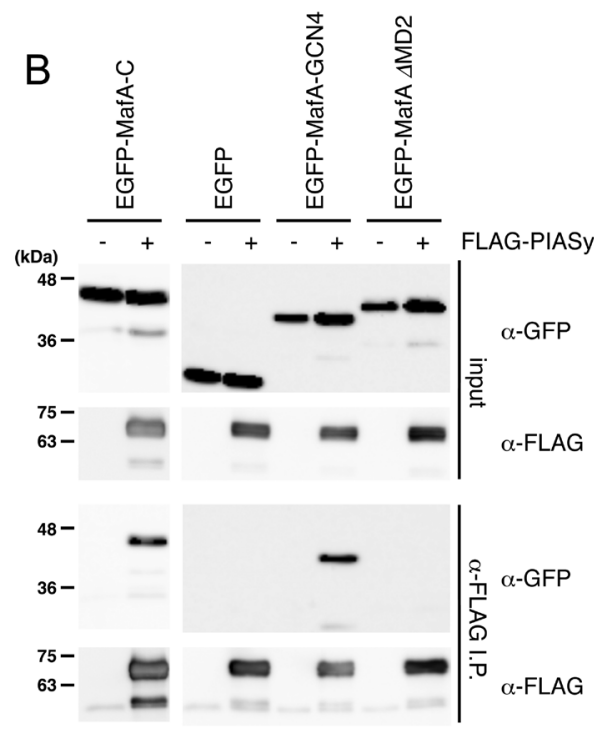

D



\section{Figure 5}

Identification of the MafA and PIASy domains required for interaction. (A) Schematic

representations of the MafA mutants used in this study. (B) HeLa cells were transfected with an expression plasmid harboring the indicated MafA mutants and with or without an expression plasmids harboring FLAG-tagged PIASy. Cell extracts were subjected to

co-immunoprecipitation using an anti-FLAG antibody, and immune complexes ( $\alpha$-FLAG I.P.) and total cell extracts (input) were analyzed by immunoblotting using anti-GFP or anti-FLAG antibodies. Data are representative of two biological replicates. (C) Schematic representations of the PIASy deletion mutants. The conserved domains (SAP, PINIT, and SP-RING) and motifs (SIM1 and SIM2) are indicated. (D) HA-tagged MafA was co-expressed with the full-length (FL) or deletion mutant of FLAG-PIASy in HeLa cells, and the cell extracts were subjected to immunoprecipitation using an anti-FLAG antibody. Immune complexes were analyzed using anti-HA or anti-FLAG antibodies. Data are representative of two independent biological replicates. strong transactivator domain of the VP16 protein from Herpes simplex virus-1 (Gal4-VP16) was also not affected by PIASy. These results suggest that PIASy does not affect the transactivator function of the amino-terminal region of MafA. Alternatively, the carboxyl-terminal region of MafA might be required for PIASy to interfere with the transactivator function of the amino-terminal region.

We further explored the regions of MafA required for PIASy-mediated repression using a luciferase reporter construct in which transcription was controlled by the rabbit $\beta$-globin promoter (RBGP) TATA-box and three tandem copies of the $\mathrm{C} 1$ element of the insulin gene promoter (pGL4/3xC1-RBGP) (Fig. 4C, upper panel). Expression of full-length (FL) MafA alone activated the reporter, and co-expression of PIASy reduced it (Fig. 4C, lower panel). The reporter was also activated by a fusion construct comprising the carboxyl-terminal DNA-binding domain (bZip region) of MafA and the transactivator domain of VP16 (VP16-MafA-bZip), and this activity was decreased by overexpression of PIASy. These results indicate that PIASy represses MafA activity through its bZip region and support the dispensability of SUMOylation for MafA repression as the bZip region of MafA does not contain the SUMOylation site (Lys32).

\section{The bZip region of MafA and the SAP domain of PIASy are required for their interaction}

To determine the region of MafA that interacts with PIASy, we constructed a fusion protein comprising EGFP and the carboxyl-terminal region of MafA containing the bZip region (EGFP-MafA-C) (Fig. 5A) and evaluated its interaction with FLAG-tagged PIASy. Immunoprecipitation using an anti-FLAG antibody and subsequent immunoblotting revealed that this fusion construct interacted with FLAG-PIASy, whereas EGFP alone did not (Fig. 5B). A chimeric mutant in which the leucine zipper and the carboxyl-terminal extremity of MafA was substituted by the leucine zipper of the yeast GCN4 protein (EGFP-MafA-GCN4) retained the ability 
to bind PIASy; however, a mutant containing a small deletion within the basic region of MafA (EGFP-MafA$\triangle \mathrm{MD} 2)$ was unable to bind to PIASy. These results suggest that MafA interacts with PIASy through the basic region.

We also examined which regions of PIASy are required for interaction with MafA using a series of FLAGtagged deletion mutants (Fig. 5C). The ability of these mutants to co-precipitate HA-MafA were determined via immunoprecipitation assays (Fig. 5D). Deletion of the carboxyl-terminal region containing two SIMs $(\Delta \mathrm{C})$ or the SP-RING domain ( $\triangle$ RING) did not affect the association PIASy with HA-MafA. By contrast, deletion of the PINIT domain ( $\triangle$ PINIT) reduced the binding affinity of PIASy for MafA, and deletion of the amino-terminal region containing the SAP domain $(\triangle \mathrm{SAP})$ abolished the binding activity completely. Therefore, PIASy interacts with MafA mainly through the amino-terminal SAP domain.

\section{The SAP domain and SIM1 are required for PIASy to repress the insulin gene promoter activity}

To further obtain mechanistic insights into the regulation of MafA by PIASy, the ability of various PIASy mutants to repress the activity of the insulin gene promoter were assessed. Synergistic activation of the insulin gene promoter reporter by MafA, Pdx1, and Beta2 in HeLa cells was decreased by expression of wild-type PIASy and the $\triangle$ RING and $\triangle$ PINIT mutants (Fig. 6A). By contrast, the PIASy $\triangle$ SAP mutant, which lacks the ability to bind MafA, did not reduce activation of the insulin gene promoter reporter. Notably, although the PIASy $\Delta \mathrm{C}$ mutant retained the ability to bind MafA, it also did not reduce the promoter activation, indicating that binding to MafA is not sufficient for the suppression of insulin gene promoter activity by PIASy. This result prompted us to examine the role of the two independent SIMs within the carboxylterminal region of PIASy. To disrupt their ability to bind SUMO, the core amino acid sequences of SIM1 (VVDL) and SIM2 (ILVL) were mutated to AAAL either alone (mutSIM1 and mutSIM2, respectively) or in combination (mutSIM1/2). The mutSIM1 and mutSIM1/2 mutants lost the ability to repress activity of the insulin gene promoter, but the mutSIM2 mutant did not, indicating that SIM1 is essential for the repressive activity of PIASy. Overall, these results suggest that binding of PIASy to MafA through the amino-terminal SAP domain is not sufficient for repression and that binding of PIASy to SUMO through the carboxyl-terminal SIM1 is also required.

Subsequently, we examined the ability of the PIASy mutants to repress the VP16-MafA fusion protein.



B
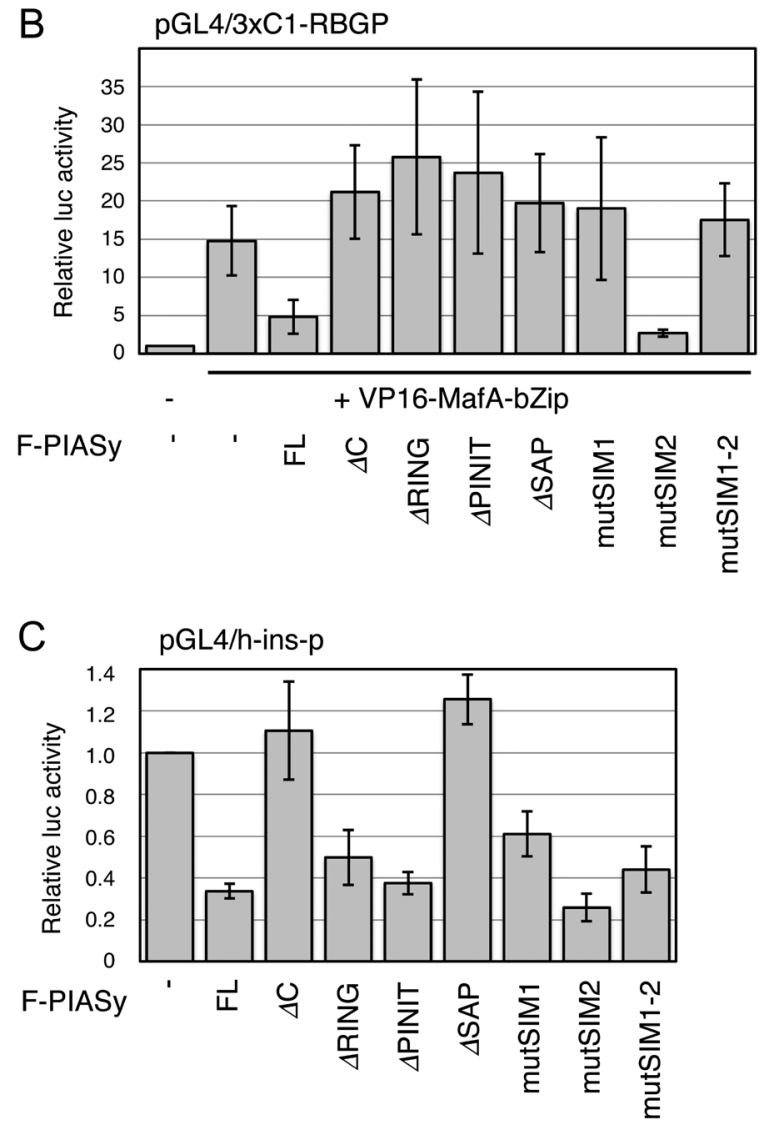

Figure 6

Requirement of the PIASy domains for transcriptional repression. (A) HeLa cells were transfected with the $\mathrm{pGL} 4 / \mathrm{h}$-ins-p reporter plasmid, expression plasmids harboring MafA, $\mathrm{Pdx} 1$, and Beta2, and expression plasmids harboring the indicated versions of PIASy. Data are expressed relative to the activity in cells transfected with $p G L 4 / h$-ins-p and the empty expression vector. Data represent the mean \pm S.E of three independent experiments. (B) HeLa cells were transfected with the pGL4/3xC1-RBGP reporter plasmid, the VP16-MafA-bZip expression plasmid, and the indicated PIASy deletion mutants. Data represent the mean \pm S.E. of three to five independent experiments. (C) NIT-1 cells were co-transfected with the $\mathrm{pGL} 4 / \mathrm{h}$-ins-p reporter plasmid and an expression plasmid harboring the indicated PIASy mutants. Data are representative of three to four independent biological replicates. 
Activation of the reporter construct containing tandem repeats of the $\mathrm{C} 1$ element (pGL4/3xC1-RBGP) by this chimeric MafA protein was inhibited by wild-type PIASy and mutSIM2 but not by any of the deletion mutants $(\Delta \mathrm{C}$, $\Delta$ RING, $\Delta$ PINIT, or $\Delta$ SAP) or by mutSIM1 or mutSIM1/2 (Fig. $6 \mathrm{~B}$ ). Although the $\triangle$ RING and $\triangle$ PINIT mutants were able to repress the synergistic activation of MafA, Pdx1, and Beta2 (Fig. 6A), they were inert in repressing VP16MafA. These findings indicate that the SP-RING and PINIT domains are also required to repress the artificial and strong transactivator VP16-MafA and suggest that PIASy has the ability to repress transcription through multiple mechanisms.

We also examined the requirement of the PIASy domains for repressing activity of the endogenous insulin gene promoter in NIT-1 cells (Fig. 6C). The $\Delta \mathrm{C}$ and $\triangle \mathrm{SAP}$ mutants were unable to reduce activity of the pGL4/h-ins-p reporter. However, similar to their ability to repress the synergistic and reconstituted activation in HeLa cells, the $\triangle$ RING and $\triangle$ PINIT mutants retained the ability to repress activity of the reporter. The mutSIM1 and mutSIM1/2 mutants were partially active in reducing reporter activity. Overall, the requirements of PIASy domains for repressing the reconstituted activity by MafA, Pdx1, and Beta2, and for repressing the endogenous activity, were similar but partially different. These findings suggest that PIASy represses activity of the insulin gene promoter mainly through MafA in $\beta$-cells, but one or more additional transcription factors are also involved in this process.

\section{Discussion}

The results presented here demonstrate that PIASy downregulates activity of the insulin gene promoter in $\beta$-cells and that PIASy physically interacts with the insulin gene transactivator MafA and represses its transcriptional activity. Previous studies showed that PIASy regulates Islet1 and Glis3 positively and negatively, respectively (Yan et al. 2016, Hoard et al. 2018). Therefore, PIASy seems to regulate the insulin promoter by interfering with multiple transcription factors including Islet1, Glis3, and MafA. However, we found that deletion of the putative Islet1-binding site A3/A4 or individual mutation of GlisBS and $\mathrm{C} 1$, the binding sites for Glis3 and MafA, respectively, did not abolish the inhibitory effect of PIASy. Further studies are needed to elucidate the detailed relationships and mechanisms of regulation among these transcription factors and PIASy.

Here, PIASy enhanced the SUMOylation of MafA, but this modification was not necessary for PIASy-mediated transcriptional repression. For example, PIASy repressed the transactivator function of a SUMOylation-deficient mutant of MafA (K32R). In addition, PIASy repressed the VP16-MafA bZip fusion protein, which lacks the SUMOylation site Lys32, but did not repress the GAL4MafA fusion protein, which contains Lys32. These observations indicate that the bZip domain of MafA is sufficient for PIASy to repress its activity. Accordingly, we found that the basic region within the bZip domain of MafA is necessary for binding to PIASy. As MafA recognizes its target DNA through the basic region, this finding led us to hypothesize that PIASy interacts with MafA to compete for binding to DNA. However, we speculate that this scenario may not be relevant, because interaction with MafA is not sufficient for PIASy to repress its transcriptional activity. For example, the $\Delta \mathrm{C}$ PIASy mutant, which retains the ability to bind MafA, was unable to repress transactivation by MafA in luciferase assays. In addition, a biotin-labeled DNA pull-down assay revealed that overexpression of PIASy did not affect binding of MafA to the $\mathrm{C} 1$ element (unpublished observation). We also found that the carboxyl-terminal SIM1 of PIASy was also necessary for its repressive activity. Therefore, we speculate that PIASy binds to MafA on the insulin gene promoter through its amino-terminal domain and inhibits transactivation through SIM1. As SIM has a SUMO-interacting function, PIASy might interact with SUMOylated transcription factors and/or co-regulators neighboring MafA on the insulin gene promoter. Such candidates may include the transcriptional co-activators p300/CBP, which are SUMOylated, and Glis3, which is able to activate the insulin gene promoter synergistically with MafA and is SUMOylated by PIASy (Yang et al. 2009, ZeRuth et al. 2013, Hoard et al. 2018). In this scenario, MafA serves as a scaffold of PIASy to repress the insulin gene promoter; to our knowledge, this is a novel mechanism of transcriptional repression by PIASy.

In contrast to PIASy-mediated repression of the endogenous or reconstituted MafA-Pdx1-Beta2 activities, PIASy also required the PINIT and SP-RING domains to repress the artificial VP16-MafA bZip protein. This unexpected finding suggests that PIASy is a multifunctional protein that interferes with multiple transcription factors and cofactors and regulates transcription through multiple mechanisms depending on its functional domains. Notably, the SP-RING domains of PIAS family members play a central role in their SUMO E3 ligase activity by interacting with the SUMO-conjugating enzyme Ubc9; however, PIASy-mediated SUMOylation of the Yin Yang1 protein does not require the SP-RING domain 
(Deng et al. 2007). The PINIT domain is reportedly required for nuclear localization of PIAS3 (Duval et al. 2003), but its molecular function is not fully understood. For some substrates, the PINIT domain plays a role in SUMOylation (Yunus \& Lima 2009). Therefore, it seems that the PINIT and SP-RING domains are required for PIASy to repress the strong transactivator VP16, in addition to the SAP and SIM domains. Further studies are required to fully characterize the molecular functions of PIAS family members.

The results of our PIASy-knockdown studies in two $\beta$-cell lines indicate that insulin gene promoter activity is not fully active in these cells, but is held in a partially repressed state by PIASy. By contrast, it has been reported that expression levels of PIASy and insulin mRNAs are upregulated in islets of $d b / d b$ mice at prediabetic 8 weeks of age (Yan et al. 2016), indicating that expression of PIASy and insulin are positively correlated in this type 2 diabetes model mice. Thus, the physiological relevance of PIASy-mediated repression and its regulation is unknown. The $\beta$-cell-enriched transcription factors, particularly MafA, Pdx1, and Beta2, play critical roles in the regulation of $\beta$-cell pathophysiology (Wilson et al. 2003, Bernardo et al. 2008), and their ectopic expression is successfully used for cellular reprogramming or lineage conversion into functional $\beta$-cells (Zhou et al. 2008, Chen et al. 2014, Furuyama et al. 2019). Understanding the functions of these transcription factors, particularly their synergistic action and regulation, including that by PIASy, will aid the development of $\beta$-cell-regenerative medicines and will contribute to the search for a cure for diabetes.

\section{Declaration of interest}

The authors declare that there is no conflict of interest that could be perceived as prejudicing the impartiality of the research reported.

\section{Funding}

This work was supported by a Grant-in-Aid for Scientific Research (C) from the Japan Society for the Promotion of Science (JSPS) (grant numbers 16K09763) to K K.

\section{Author contribution statement}

Suzuka Onishi performed most of the experiments and analyzed the data. Kohsuke Kataoka designed the research study, performed some of the experiments, and wrote the manuscript.

\section{Acknowledgements}

The authors are grateful to Jun-ichi Miyazaki (Osaka University) for the MIN6 cell line. They are indebted to Dr Tetsuro Kokubo for helpful discussions.

\section{References}

Aramata S, Han SI, Yasuda K \& Kataoka K 2005 Synergistic activation of the insulin gene promoter by the $\beta$-cell enriched transcription factors MafA, Beta2, and Pdx1. Biochimica et Biophysica Acta 1730 41-46. (https://doi.org/10.1016/j.bbaexp.2005.05.009)

Artner I, Hang Y, Mazur M, Yamamoto T, Guo M, Lindner J, Magnuson MA \& Stein R 2010 MafA and MafB regulate genes critical to $\beta$-cells in a unique temporal manner. Diabetes 59 2530-2539. (https://doi.org/10.2337/db10-0190)

Bernardo AS, Hay CW \& Docherty K 2008 Pancreatic transcription factors and their role in the birth, life and survival of the pancreatic $\beta$ cell. Molecular and Cellular Endocrinology 294 1-9. (https://doi. org/10.1016/j.mce.2008.07.006)

Brand AH \& Perrimon N 1993 Targeted gene expression as a means of altering cell fates and generating dominant phenotypes. Development 118 401-415.

Butler AE, Robertson RP, Hernandez R, Matveyenko AV, Gurlo T \& Butler PC 2012 Beta cell nuclear musculoaponeurotic fibrosarcoma oncogene family A (MafA) is deficient in type 2 diabetes. Diabetologia 55 2985-2988. (https://doi.org/10.1007/s00125-012-2666-2)

Chen YJ, Finkbeiner SR, Weinblatt D, Emmett MJ, Tameire F, Yousefi M, Yang C, Maehr R, Zhou Q, Shemer R, et al. 2014 De novo formation of insulin-producing 'neo- $\beta$ cell islets' from intestinal crypts. Cell Reports 6 1046-1058. (https://doi.org/10.1016/j.celrep.2014.02.013)

Deng Z, Wan M \& Sui G 2007 PIASy-mediated sumoylation of Yin Yang 1 depends on their interaction but not the RING finger. Molecular and Cellular Biology 27 3780-3792. (https://doi.org/10.1128/ MCB.01761-06)

Docherty HM, Hay CW, Ferguson LA, Barrow J, Durward E \& Docherty K 2005 Relative contribution of PDX-1, MafA and E47/ $\beta 2$ to the regulation of the human insulin promoter. Biochemical Journal 389 813-820. (https://doi.org/10.1042/BJ20041891)

Duval D, Duval G, Kedinger C, Poch O \& Boeuf H 2003 The 'PINIT' motif, of a newly identified conserved domain of the PIAS protein family, is essential for nuclear retention of PIAS3L. FEBS Letters 554 111-118. (https://doi.org/10.1016/S0014-5793(03)01116-5)

Fujitani Y, Fujitani S, Boyer DF, Gannon M, Kawaguchi Y, Ray M, Shiota M, Stein RW, Magnuson MA \& Wright CVE 2006 Targeted deletion of a cis-regulatory region reveals differential gene dosage requirements for $\mathrm{Pdx} 1$ in foregut organ differentiation and pancreas formation. Genes and Development 20 253-266. (https://doi. org/10.1101/gad.1360106)

Furuyama K, Chera S, van Gurp L, Oropeza D, Ghila L, Damond N, Vethe H, Paulo JA, Joosten AM, Berney T, et al. 2019 Diabetes relief in mice by glucose-sensing insulin-secreting human $\alpha$-cells. Nature 567 43-48. (https://doi.org/10.1038/s41586-019-0942-8)

Gross M, Yang R, Top I, Gasper C \& Shuai K 2004 PIASy-mediated repression of the androgen receptor is independent of sumoylation. Oncogene 23 3059-3066. (https://doi.org/10.1038/sj.onc.1207443)

Gu C, Stein GH, Pan N, Goebbels S, Hörnberg H, Nave KA, Herrera P, White P, Kaestner KH, Sussel L, et al. 2010 Pancreatic $\beta$ cells require NeuroD to achieve and maintain functional maturity. Cell Metabolism 11 298-310. (https://doi.org/10.1016/j. cmet.2010.03.006)

Guo S, Dai C, Guo M, Taylor B, Harmon JS, Sander M, Robertson RP, Powers AC \& Stein R 2013 Inactivation of specific $\beta$ cell transcription factors in type 2 diabetes. Journal of Clinical Investigation 123 33053316. (https://doi.org/10.1172/JCI65390)

Han SI, Aramata S, Yasuda K \& Kataoka K 2007 MafA stability in pancreatic $\beta$-cells is regulated by glucose and is dependent on its constitutive phosphorylation at multiple sites by glycogen synthase kinase 3. Molecular and Cellular Biology 27 6593-6605. (https://doi. org/10.1128/MCB.01573-06)

Han SI, Yasuda K \& Kataoka K 2011 ATF2 interacts with $\beta$-cell-enriched transcription factors, MafA, Pdx1, and Beta2, and activates insulin 
gene transcription. Journal of Biological Chemistry 286 10449-10456. (https://doi.org/10.1074/jbc.M110.209510)

Han SI, Tsunekage Y \& Kataoka K 2016 Phosphorylation of MafA enhances interaction with Beta2/NeuroD1. Acta Diabetologica 53 651-660. (https://doi.org/10.1007/s00592-016-0853-1)

Harmon JS, Stein R \& Robertson RP 2005 Oxidative stress-mediated, post-translational loss of MafA protein as a contributing mechanism to loss of insulin gene expression in glucotoxic beta cells. Journal of Biological Chemistry 280 11107-11113. (https://doi.org/10.1074/jbc. M410345200)

Hoard TM, Yang XP, Jetten AM \& ZeRuth GT 2018 PIAS-family proteins negatively regulate Glis3 transactivation function through SUMO modification in pancreatic $\beta$ cells. Heliyon 4 e00709. (https://doi. org/10.1016/j.heliyon.2018.e00709)

Joazeiro CAP \& Weissman AM 2000 RING finger proteins: mediators of ubiquitin ligase activity. Cell 102 549-552. (https://doi.org/10.1016/ S0092-8674(00)00077-5)

Jung Y, Zhou R, Kato T, Usui JK, Muratani M, Oishi H, Heck MMS \& Takahashi S 2018 Isl1 $\beta$ overexpression with key $\beta$ cell transcription factors enhances glucose-responsive hepatic insulin production and secretion. Endocrinology 159 869-882. (https://doi.org/10.1210/ en.2017-00663)

Kanai K, Reza HM, Kamitani A, Hamazaki Y, Han SI, Yasuda K \& Kataoka K 2010 SUMOylation negatively regulates transcriptional and oncogenic activities of MafA. Genes to Cells 15 971-982. (https:// doi.org/10.1111/j.1365-2443.2010.01431.x)

Kanai K, Aramata S, Katakami S, Yasuda K \& Kataoka K 2011 Proteasome activator PA28 $\gamma$ stimulates degradation of GSK3-phosphorylated insulin transcription activator MAFA. Journal of Molecular Endocrinology 47 119-127. (https://doi.org/10.1530/JME-11-0044)

Kaneto H, Matsuoka TA, Nakatani Y, Miyatsuka T, Matsuhisa M, Hori M \& Yamasaki Y 2005 A crucial role of MafA as a novel therapeutic target for diabetes. Journal of Biological Chemistry 280 15047-15052. (https://doi.org/10.1074/jbc.M412013200)

Kataoka K, Yoshitomo-Nakagawa K, Shioda S \& Nishizawa M 2001a A set of Hox proteins interact with the Maf oncoprotein to inhibit its DNA binding, transactivation, and transforming activities. Journal of Biological Chemistry 276 819-826. (https://doi.org/10.1074/jbc. M007643200)

Kataoka K, Shioda S, Yoshitomo-Nakagawa K, Handa H \& Nishizawa M $2001 b$ Maf and jun nuclear oncoproteins share downstream target genes for inducing cell transformation. Journal of Biological Chemistry 276 36849-36856. (https://doi.org/10.1074/jbc.M102234200)

Kataoka K, Han SI, Shioda S, Hirai M, Nishizawa M \& Handa H 2002 MafA is a glucose-regulated and pancreatic $\beta$-cell-specific transcriptional activator for the insulin gene. Journal of Biological Chemistry 277 49903-49910. (https://doi.org/10.1074/jbc. M206796200)

Kaur K, Park H, Pandey N, Azuma Y \& De Guzman RN 2017 Identification of a new small ubiquitin-like modifier (SUMO)interacting motif in the E3 ligase PIASy. Journal of Biological Chemistry 292 10230-10238. (https://doi.org/10.1074/jbc. M117.789982)

Long J, Matsuura I, He D, Wang G, Shuai K \& Liu F 2003 Repression of Smad transcriptional activity by PIASy, an inhibitor of activated stat. PNAS 100 9791-9796. (https://doi.org/10.1073/pnas.1733973100)

Malecki MT, Jhala US, Antonellis A, Fields L, Doria A, Orban T, Saad M, Warram JH, Montminy M \& Krolewski AS 1999 Mutations in NEUROD1 are associated with the development of type 2 diabetes mellitus. Nature Genetics 23 323-328. (https://doi.org/10.1038/15500)
Miyazaki JI, Araki K, Yamato E, Ikegami H, Asano T, Shibasaki Y, Oka Y \& Yamamura KI 1990 Establishment of a pancreatic $\beta$ cell line that retains glucose-inducible insulin secretion: special reference to expression of glucose transporter isoforms. Endocrinology 127 126132. (https://doi.org/10.1210/endo-127-1-126)

Naya FJ, Huang HP, Qiu Y, Mutoh H, DeMayo FJ, Leiter AB \& Tsai MJ 1997 Diabetes, defective pancreatic morphogenesis, and abnormal enteroendocrine differentiation in BETA2/NeuroD-deficient mice. Genes and Development 11 2323-2334. (https://doi.org/10.1101/ gad.11.18.2323)

Nishimura W, Kondo T, Salameh T, El Khattabi I, Dodge R, BonnerWeir S \& Sharma A 2006 A switch from MafB to MafA expression accompanies differentiation to pancreatic $\beta$-cells. Developmental Biology 293 526-539. (https://doi.org/10.1016/j.ydbio.2006.02.028)

Robertson RP 2004 Chronic oxidative stress as a central mechanism for glucose toxicity in pancreatic islet beta cells in diabetes. Journal of Biological Chemistry 279 42351-42354. (https://doi.org/10.1074/jbc. R400019200)

Rytinki MM, Kaikkonen S, Pehkonen P, Jääskeläinen T \& Palvimo JJ 2009 PIAS proteins: pleiotropic interactors associated with SUMO. Cellular and Molecular Life Sciences 66 3029-3041. (https://doi. org/10.1007/s00018-009-0061-z)

Sachdev S, Bruhn L, Sieber H, Pichler A, Melchior F \& Grosschedl R 2001 PIASy, a nuclear matrix-associated SUMO E3 ligase, represses LEF1 activity by sequestration into nuclear bodies. Genes and Development 15 3088-3103. (https://doi.org/10.1101/gad.944801)

Shao C \& Cobb MH 2009 SUMOylation regulates the transcriptional activity of MafA in pancreatic $\beta$ cells. Journal of Biological Chemistry 284 3117-3124. (https://doi.org/10.1074/jbc.M806286200)

Stoffers DA, Stanojevic V \& Habener JF 1998 Insulin promoter factor-1 gene mutation linked to early-onset type 2 diabetes mellitus directs expression of a dominant negative isoprotein. Journal of Clinical Investigation 102 232-241. (https://doi.org/10.1172/JCI2242)

Wilson ME, Scheel D \& German MS 2003 Gene expression cascades in pancreatic development. Mechanisms of Development 120 65-80. (https://doi.org/10.1016/S0925-4773(02)00333-7)

Yan C, Yu C, Zhang D, Cui Y, Zhou J \& Cui S 2016 Protein inhibitor of activated STAT Y (PIASy) regulates insulin secretion by interacting with LIM homeodomain transcription factor Isl1. Scientific Reports 6 39308. (https://doi.org/10.1038/srep39308)

Yang Y, Chang BHJ, Samson SL, Li MV. \& Chan L 2009 The Krüppel-like zinc finger protein Glis3 directly and indirectly activates insulin gene transcription. Nucleic Acids Research 37 2529-2538. (https://doi. org/10.1093/nar/gkp122)

Yunus AA \& Lima CD 2009 Structure of the Siz/PIAS SUMO E3 ligase Siz1 and determinants required for SUMO modification of PCNA. Molecular Cell 35 669-682. (https://doi.org/10.1016/j. molcel.2009.07.013)

ZeRuth GT, Takeda Y \& Jetten AM 2013 The Krüppel-like protein Glisimilar 3 (Glis3) functions as a key regulator of insulin transcription. Molecular Endocrinology 27 1692-1705. (https://doi.org/10.1210/ me.2013-1117)

Zhang H, Wang WP, Guo T, Yang JC, Chen P, Ma KT, Guan YF \& Zhou CY 2009 The LIM-homeodomain protein ISL1 activates insulin gene promoter directly through synergy with BETA2. Journal of Molecular Biology 392 566-577. (https://doi.org/10.1016/j. jmb.2009.07.036)

Zhou Q, Brown J, Kanarek A, Rajagopal J \& Melton DA 2008 In vivo reprogramming of adult pancreatic exocrine cells to $\beta$-cells. Nature $\mathbf{4 5 5}$ 627-632. (https://doi.org/10.1038/nature07314) https://jme.bioscientifica.com

https://doi.org/10.1530/JME-19-0172 (c) 2019 Society for Endocrinology Published by Bioscientifica Ltd. Printed in Great Britain
Received in final form 18 September 2019

Accepted 14 October 2019

Accepted Preprint published online 14 October 2019 